\title{
Comparison of modified corneal cross-linking with intrastromal voriconazole for the treatment of fungal corneal ulcer
}

\author{
YINGXIN CHEN $^{1}$, XINGYA MIAO ${ }^{1}$, MINGHONG GAO $^{1}$ and LIXIN SONG ${ }^{2}$ \\ Departments of ${ }^{1}$ Ophthalmology and ${ }^{2}$ Dermatology, General Hospital of Northern Theater Command, \\ Shenyang, Liaoning 110016, P.R. China
}

Received June 11, 2020; Accepted March 1, 2021

DOI: $10.3892 /$ etm.2021.10218

\begin{abstract}
The present study aimed to evaluate the efficacy of modified corneal cross-linking (CXL) for the treatment of fungal corneal ulcers compared with that following intrastromal voriconazole injection. In total, 31 patients with fungal corneal ulcers treated at The General Hospital of Northern Theater Command between October 2017 and October 2019 were enrolled. Among them, 10 eyes were treated with ultraviolet A (UV-A)/riboflavin CXL (CXL group), whilst 21 eyes were treated with debridement combined with intrastromal voriconazole (stromal injection group). Preoperative microbiological examination was performed in both groups, and evaluated using Fisher's exact test. Postoperatively, infection control and total efficacy rates, localized lesion, ulcer healing rate 1 week after surgery, visual acuity and complications were evaluated using Fisher's exact test, however visual acuity was analyzed by mixed-model ANOVA. The results showed that the pre-operative species distribution between the CXL and stromal injection groups did not significantly differ. The infection control rate in the CXL group was notably higher compared with that in the stromal injection group $(\mathrm{P}=0.04)$. Furthermore, the total efficacy rate in the CXL group was also markedly higher compared with that in the stromal injection group, though no statistically significant differences were observed. Localized lesions were observed in nine eyes (90.0\%) in the CXL group and nine eyes (42.9\%) in the stromal injection group $(\mathrm{P}=0.02)$. However, the rate of ulcer healing at 1 week postoperatively and the logarithm of the minimum angle of resolution ( $\log \mathrm{MAR})$ of visual acuity were not found to be significantly different between the two groups. In terms of complications, with the exception of one patient in the CXL group exhibiting loss of corneal transparency and one patient
\end{abstract}

Correspondence to: Professor Lixin Song, Department of Dermatology, General Hospital of Northern Theater Command, 83 Wenhua Road, Shenyang, Liaoning 110016, P.R. China E-mail: songlixin2020@163.com

Key words: corneal cross-linking, debridement combined with intrastromal voriconazole injection, fungal corneal ulcer, fungal keratitis, visual acuity in the stromal injection group presenting with partial corneal thinning, no other forms of complications were observed. In conclusion, the present study suggested that CXL could have a beneficial impact for treating fungal corneal ulcers in the aspects of infection control, localized lesions and accelerated epithelialization. In addition, except the loss of corneal transparency, this treatment approach could be applied with reduced risks of adverse events.

\section{Introduction}

Fungal keratitis (FK) is a devastating corneal disorder, with $\sim 40 \%$ prevalence of infectious keratitis prevalence in China, which is mainly associated with agricultural trauma $(1,2)$. Once infected, corneal antigenicity changes and the immune system is activated, resulting in increased inflammatory cell infiltration, which release a large number of inflammatory factors (3). FK remains to be the major cause of fungal corneal ulcers in northern China (4). At present, antifungal medications and surgery are the main treatment approaches for fungal corneal ulcer (5). However, several problems remain, including the emergence of drug-resistant strains of pathogens, long treatment cycles, recurrence, high treatment costs and complications such as hepatorenal function impairment and rejection (6). Therefore, development of novel and effective treatment strategies for fungal corneal ulcer is urgently required.

Corneal cross-linking (CXL) is a photochemical reaction mediated by ultraviolet A (UV-A) radiation and the photosensitizer riboflavin $(7,8)$. Previous studies have demonstrated that CXL is effective against bacterial and fungal corneal ulcers $(9,10)$. The traditional CXL procedure involves irradiation of the eye at $3 \mathrm{~mW} / \mathrm{cm}_{2}$ for $30 \mathrm{~min}$ using a total energy dose of $5.4 \mathrm{~J} / \mathrm{cm}^{2}$ (7). However, certain limitations remain for the clinical application of CXL, including long irradiation time, limited dose of irradiation and severe corneal irritation $(11,12)$. Currently, although new CXL technologies have been developed to shorten the operation time and improve the comfort level of patients postoperatively, their effects, including localized lesion and infection control, remain unsatisfactory compared with those following traditional CXL (13-15). Therefore, irradiation time and total irradiation dose was improved according to the principle of the biological efficacy associated with total energy dose (16). A similar study 
indicated that increased irradiation at $7-45 \mathrm{~mW} / \mathrm{cm}^{2}$ shortened irradiation time, whilst a total energy dose of $7.2 \mathrm{~J} / \mathrm{cm}^{2}$ was also reasonable and safe (17). Furthermore, Özdemir et al (18) demonstrated that CXL combined with voriconazole exhibited beneficial effects in a rabbit model of fungal keratitis. However, the effect of CXL compared with that of intrastromal voriconazole injection for treating fungal corneal ulcers remains unclear.

The present study aimed to evaluate the efficiency of a modified CXL procedure, which involves irradiation at $30 \mathrm{~mW} / \mathrm{cm}^{2}$ for $4 \mathrm{~min}$ using a total energy dose of $7.2 \mathrm{~J} / \mathrm{cm}^{2}$, compared with that of intrastromal voriconazole injection. The findings of the present study could reveal a novel therapeutic option for treating fungal corneal ulcer.

\section{Materials and methods}

Patients. In the present study, 31 patients (sex, 19 males and 12 females; age, 55.3 \pm 9.0 years) with fungal corneal ulcer who were treated at the General Hospital of Northern Theater Command (Shenyang, China) between October 2017 and October 2019 were enrolled.

The inclusion criteria were as follows: i) Patients diagnosed with fungal corneal ulcer by corneal scraping, fungal culture or in vivo confocal microscopy (IVCM; Figs. 1 and 2); ii) the ulcer depth did not exceed the $2 / 3$ of the corneal thickness, as measured using slit lamp or anterior segment optical coherence tomography (AS-OCT); iii) patients did not heal despite conservative therapy for $\geq 7$ days; iv) no indication for emergency surgery; v) patients cooperated with eye examination and aged from 18 to 90 years; and vi) followed up for $\geq 6$ months. Exclusion criteria were as follows: i) Patients with systemic diseases that could potentially influence vision, including diabetes, hypertension and cardiovascular diseases; ii) lesions with full-thickness or even perforated, observation of obvious endothelial plaques and anterior chamber empyema; and iii) with fundus lesions, including fundus hemorrhage, macular degeneration and optic nerve atrophy, which affected postoperative vision in the past.

According to the surgical approach, patients who were treated with CXL were defined as the CXL group $(n=10)$, whilst patients with corneal debridement combined with intrastromal voriconazole injection were defined as the stromal injection group $(n=21)$. The advantages and disadvantages of two treatment methods were addressed for each patient, following which a surgical plan was developed according to the patients' choice. In addition, some patients did not accept CXL due to economic conditions. Therefore, the differences in sample size between the two groups appear to be large. The present study was approved by the Ethics Committee of the General Hospital of Northern Theater Command (approval no. 201736). All eligible patients provided written informed consent prior to treatment and all operations were completed by Dr MG.

Microbiological examination. Specimens were collected from the surface of corneal ulcer, and then cultured on Sabouraud agar medium at $28^{\circ} \mathrm{C}$ for 1-7 days (cat. no. YC-SDA-90; Shenyang Yancheng Biological Products Co., Ltd.). Species or genus was identified by colony morphology referring to
Manual of Clinical Microbiology, 11th Edition (19). For filamentous fungus that cannot be identified by morphology, $18 \mathrm{~S}$ rRNA or ITS sequencing was conducted by BGI Genomics.

Surgical procedures. In the CXL group, patients were treated two or three times with pilocarpine nitrate for $10 \mathrm{~min}$ prior surgery. Subsequently, local anesthetic was administered before the conjunctival sac and surface of lesions were washed with $0.9 \% \mathrm{NaCl}$. Following removal of the epithelium and necrotic tissue, a corneal ring was placed on the surface of the cornea and $0.1 \%$ of riboflavin (Avedro Inc.) was instilled every $5 \mathrm{~min}$ for $30 \mathrm{~min}$. Until riboflavin entered the anterior chamber, the cornea was irradiated for 4 min using a KXL I UV-A source (Avedro Inc.) at a wavelength of $370 \mathrm{~nm}$, beam diameter of $9 \mathrm{~mm}$, irradiance of $30 \mathrm{~mW} / \mathrm{cm}^{2}$ with a total energy dose of $7.2 \mathrm{~J} / \mathrm{cm}^{2}$. Following irradiation, ofloxacin ointment (Shenyang Xingqi Pharmaceutical Co., Ltd.) was applied to the eyes and sterile auxiliary materials were used to cover them.

For patients in the stromal injection group, local infiltration anesthesia was performed around the eyeball using $0.5 \%$ proparacaine hydrochloride (Alcaine, Alcon Laboratories, Inc.). Subsequently, the conjunctival sac and surface of the lesions were washed for two to three times with $0.9 \% \mathrm{NaCl}$ before the lesion necrotic tissue was removed without exceeding $1 / 2$ of the corneal thickness. A 1-ml syringe attached to a $30 \mathrm{G}$ needle was then used to penetrate the corneal stroma at a relatively horizontal angle from the transparent cornea area of the lesion edge. Voriconazole (0.5 mg/ml; Sichuan Meidakang Pharmaceutical Co., Ltd.) was slowly injected to form an edema before the extent of corneal edema was used to evaluate the coverage of the drug. The injection dose of voriconazole depended on the size of the lesion. In general, the infiltration area of voriconazole was larger than the lesion coverage by $0.5 \mathrm{~mm}$. The injection was repeated after 3-5 days if lesions were not localized or were deepened. Following surgery, ofloxacin ointment was applied to the eyes, which were finally covered with sterile auxiliary materials.

Postoperative management. Antibiotic therapy was administrated after the surgical procedure. Briefly, the eyes were treated postoperatively with $5 \%$ natamycin eye drops (four to six times/day; one drop each time), $0.5 \%$ levofloxacin eye drops (twice/day; one drop each time) and $0.3 \%$ sodium hyaluronate eye drops (four times/day; one drop each time), $3 \%$ ofloxacin eye ointment (once/day; one drop) and oral fluconazole capsules $(0.2 \mathrm{~g}$; first dosage, $0.4 \mathrm{~g})$. The aforementioned therapies were administrated for $\geq 4$ weeks. If the lesions were not improved or worsen, lamellar corneal transplant surgery would be performed. The follow-up of all cases lasted for $\geq 6$ months.

Postoperative evaluation indices. Treatment efficacy was evaluated according to the following efficacy evaluation criteria (20): i) 'Cured' was defined as healed ulcer and no fungal hyphae; ii) 'effective' treatment was defined as partially healed ulcers, alleviated inflammatory responses, relieved lesions and no fungal hyphae or reduction in hyphae, or treatment being effective at first, followed by reoccurrence of infection and wound not healing; and iii) 'ineffective' treatment was defined 

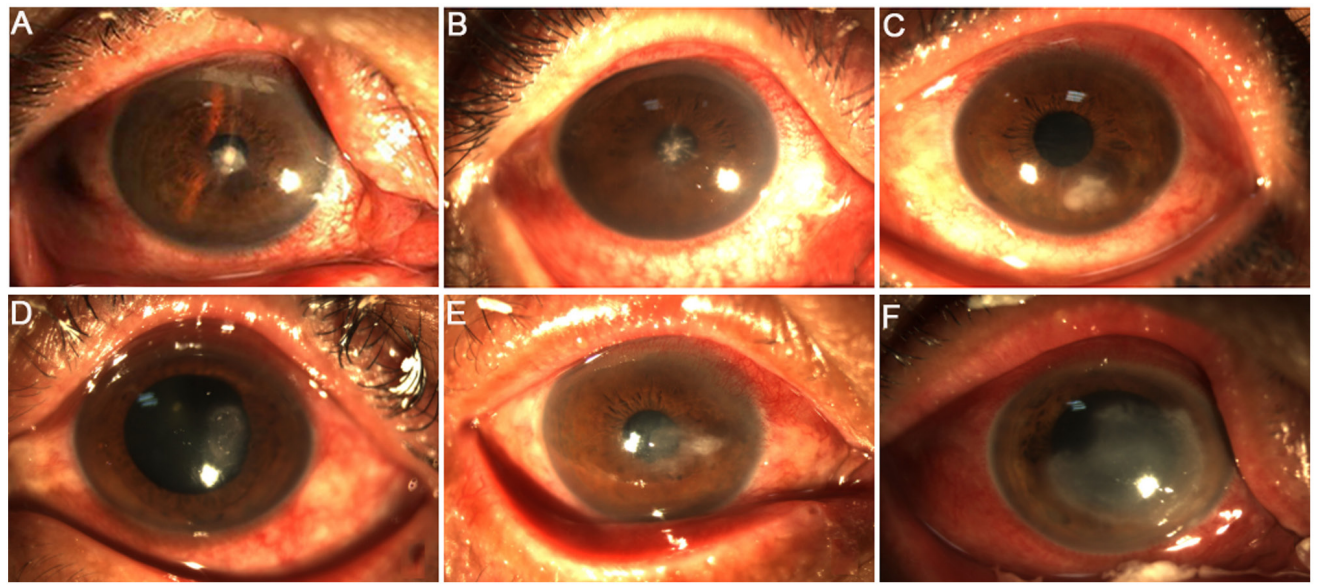

Figure 1. Representative images of the preoperative lesions. (A) Patient with lesion coverage $\leq 5 \mathrm{~mm}$, and lesion was seen in the center of the cornea with moss-like structures. (B) Lesion was seen in the center of the cornea with peripheral pseudopod and satellite foci. (C) Lesion was located at 5 o'clock position between the pupil and the limbus with unclear borders. (D) Lesion was located at 3 o'clock position in the cornea. (E) Lesion coverage was $>5 \mathrm{~mm}$, and round lesion was seen with moss-like structures and unclear borders. (F) Irregular lesion was seen with cell infiltration reaching the stromal layer.

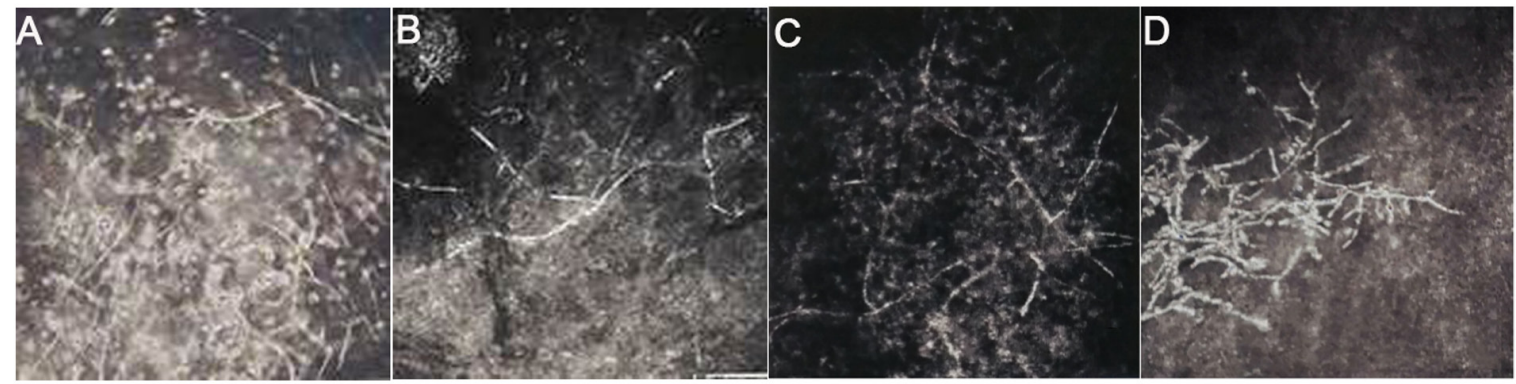

Figure 2. Representative images of fungal mycelia in lesions prior to surgery. (A) A 56-year-old female patient, (B) 61-year-old male patient, (C) 55-year-old male patient and (D) 60-year-old female patient all showed the hyphae structure of typical fungus in the lesion using in vivo confocal microscopy (magnification, $\mathrm{x} 400$ ).

by increased numbers of fungal hyphae, aggravated area and depth of ulcer. Infection control and total efficacy rates were calculated according to the following equations: i) Infection control rate $(\%)=$ cured cases/total cases $\times 100$; ii) total effective rate $(\%)=($ cured cases + effective cases $) /$ total cases $x 100$.

The definition of localized lesions was that the area and depth of ulcer did not increase or decrease and the inflammation was reduced, as previously described (21). IVCM was used to evaluate the structure of corneal lesions, fungal hyphae, infection depth, cell morphology and arrangement. In addition, 1 week after surgery, sodium fluorescein eye detection test paper (Tianjin Jingming New Technology Development Co., Ltd.) was applied to stain the cornea as previously described (22). No staining of the cornea would indicate that the ulcer was cured. Ulcer healing rate was calculated according to the following equation: Ulcer healing rate $(\%)=$ cured cases/total cases $\times 100$. Furthermore, the best corrected visual acuity (BCVA) was first determined, which was then converted to logarithm of minimal angle of resolution ( $\log$ MAR) to evaluate the outcome of the surgery. AS-OCT was used to evaluate the postoperative corneal parameters included corneal thickness, stromal infiltration and corneal epithelium healing.

Complications. In the present study, the incidence of complications, including corneal epithelial haze, loss of corneal transparency, corneal melting, viral keratitis or iritis and other types of eye tissue damage were recorded at postoperative 1 , $2,4,12$ and 24 weeks.

Statistical analysis. All data were analyzed using the SPSS 22.0 software (IBM Corp.). BCVA, expressed as the logMAR, was analyzed using the Mann-Whitney $\mathrm{U}$ test. The ages between the groups were compared with a Student's t-test, whilst the enumeration data, including sex, pathogenesis, lesion coverage, infection control, total effective and ulcer healing rates were analyzed by Fisher's exact test. Mixed-model ANOVA was used to analyze the effects of between-groups, within-group and interaction of group and time on BCVA. If the time or interaction effects were significant, Bonferroni-adjusted post hoc analysis was done to assess the comparison. $\mathrm{P}<0.05$ was considered to indicate a statistically significant difference.

\section{Results}

General characteristics. The baseline characteristics of the patients in the CXL and stromal injection groups are shown in Table I. There were no significant differences in age, sex, pathogenesis, lesion coverage and BCVA between the two groups.

Efficacy evaluation after surgery. The infection control rates in the CXL and stromal injection groups were 90.0 (9/10 eyes) 
Table I. General characteristics of patients in the two treatment groups.

\begin{tabular}{lcccc}
\hline Parameter & CXL $(\mathrm{n}=10)$ & Stromal injection $(\mathrm{n}=21)$ & Z/t/ $\chi^{2}$ value & P-value \\
\hline Age, years & $56.4 \pm 6.5$ & $54.7 \pm 10.1$ & -0.48 & 0.63 \\
Sex (male/female), $\mathrm{n}(\%)$ & $7 / 3(70.0 / 30.0)$ & $12 / 9(57.1 / 42.9)$ & 0.47 & 0.70 \\
Pathogenesis, $\mathrm{n}(\%)$ & & & 1.75 & 0.83 \\
Trauma history & $8(80.0)$ & $16(76.2)$ & & \\
History of medication and surgery & $2(20.0)$ & $2(9.5)$ & & $>0.99$ \\
Other & $0(0.0)$ & $3(14.3)$ & & \\
Lesion coverage, $\mathrm{n}(\%)$ & $9(90.0)$ & $17(81.0)$ & & \\
$\leq 5$ mm & $1(10.0)$ & $4(19.0)$ & & \\
$>5$ mm & $1.28 \pm 1.08$ & $1.62 \pm 1.33$ & -0.47 & \\
BCVA (logMAR) & & & \\
\hline
\end{tabular}
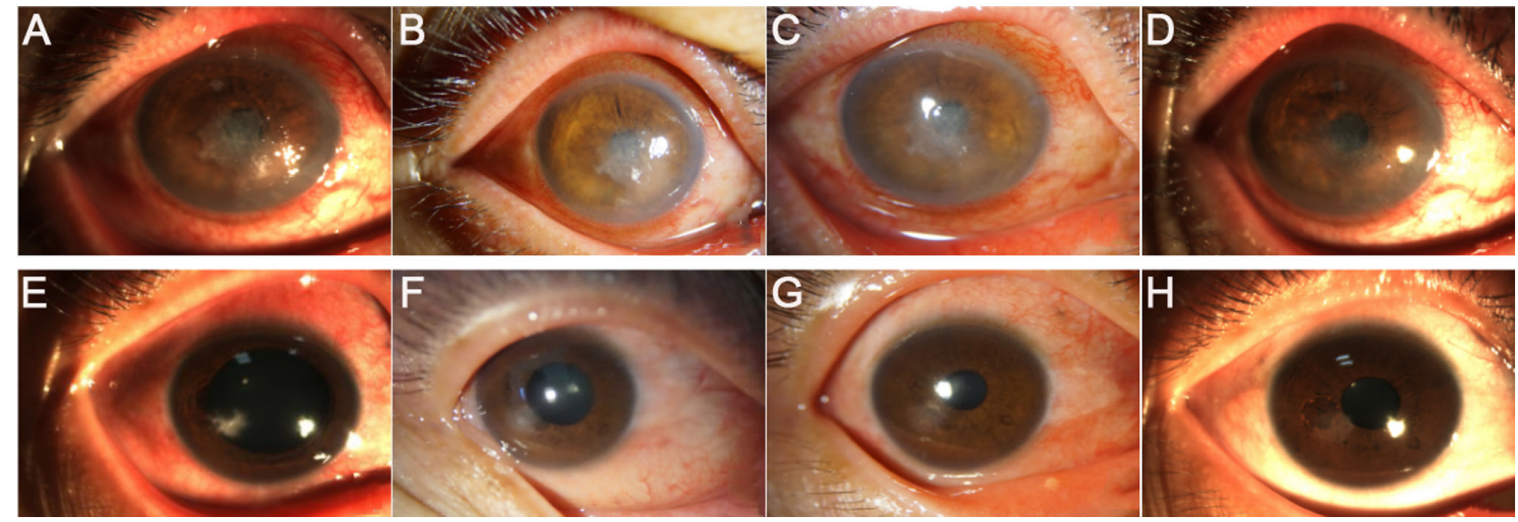

Figure 3. Representative images of cases of controlled infection. Images of a patient's ulcers (A) prior to surgery and at (B) 1 , (C) 4 and (D) 12 weeks after surgery in the corneal cross-linking group. A patient's images of ulcers (E) prior to surgery and at (F) 1, (G) 4 and (H) 12 weeks after surgery in the stromal injection group.

and $47.6 \%$ (10/21 eyes), respectively ( $\mathrm{P}=0.04$; Table II), whilst the total effective rate was $90.0 \%$ (9/10 eyes) for the CXL and $80.9 \%$ (17/21 eyes) for the stromal injection group (Table III). However, 7 eyes (33.3\%) in the stromal injection group were temporarily relieved 1 week after surgery, infection recurred and the wound was not healed between weeks 2-4, which belonged to effective group (Table III). To treat these fungal corneal ulcers further, corneal transplantation was performed. At 4 weeks after surgery, lesion was localized and the epithelium was healed in the CXL group, where the corneal transparency was increased at 12 weeks after surgery (Fig. 3). Similarly, in the stromal injection group, the border of the corneal ulcer was localized, which was cleared 1 week after the operation (Fig. 3).

Localized lesion. The localized lesions in both groups at 1 , 2, 4, 12 and 24 weeks after surgery were shown in Table IV. Localized lesions were observed in nine eyes $(90.0 \%)$ in the CXL group and nine eyes $(42.9 \%)$ in the stromal injection group 4, 12 and 24 weeks after surgery $(\mathrm{P}=0.02)$. These findings suggest that treatment with CXL was superior compared with intrastromal voriconazole in the aspect of localized lesions. Furthermore, the eyes were examined for focal lesions before and after surgery in the CXL group. The examination revealed that an ulcer area of $2 \times 2 \mathrm{~mm}$ was present in the center of the cornea preoperatively. In addition, pseudopods and satellite foci were also present (Fig. 4A). At 3, 5 and 14 days after surgery, the ulcer surface became more diffused with poorly defined borders, where the pseudopods and satellite foci disappeared (Fig. 4B-D). Furthermore, a large number of fungal mycelia, defects in the epithelial tissue, irregular cell morphology and inflammatory cell infiltration was observed in the shallow stromal layer before operation using IVCM (Fig. 5A-D). At 4 weeks following surgery, the mycelium-like structures in the shallow stromal layer disappeared and 'honeycomb'-like structures were visible (Fig. 5E). However, the number of honeycomb structures in the deep stromal layer was decreased compared with that of 4 weeks after surgery (Fig. 5G). Additionally, the number of inflammatory cells was reduced, where some scar tissues were formed (Fig. 5F) and endothelial cells were regular with normal morphology and arrangement (Fig. 5H).

Ulcer healing after surgery. The ulcer healing rate 1 week after surgery in the CXL and stromal injection groups was 60.0 (6/10 eyes) and 23.8\% (5/21 eyes), respectively. However, no statistically significant difference between the two groups were observed $(\mathrm{P}=0.11$; Table $\mathrm{V})$. The degree of ulcer healing following treatment with UV A-riboflavin CXL is shown in Fig. 6. Prior to surgery, the cornea was extensively stained 
Table II. Comparison of infection control between the two groups.

\begin{tabular}{|c|c|c|c|}
\hline Infection control & CXL $(n=10)$ & Stromal injection $(\mathrm{n}=21)$ & P-value \\
\hline Controlled infection, $\mathrm{n}(\%)$ & $9(90.0)$ & $10(47.6 \%)$ & \\
\hline Uncontrolled infection, $\mathrm{n}(\%)$ & $1(10.0)$ & $11(52.4 \%)$ & 0.04 \\
\hline
\end{tabular}

CXL, corneal cross-linking.

Table III. Comparison of treatment efficacy between the two groups.

\begin{tabular}{lcc}
\hline Treatment outcome & CXL $(\mathrm{n}=10)$ & Stromal injection $(\mathrm{n}=21)$ \\
\hline Cured, $\mathrm{n}(\%)$ & $9(90.0)$ & $10(47.6)$ \\
Effective, $\mathrm{n}(\%)$ & $0(0.0)$ & $7(33.3)$ \\
Ineffective, $\mathrm{n}(\%)$ & $1(10.0)$ & $4(19.1)$ \\
\hline
\end{tabular}

CXL, corneal cross-linking.

Table IV. Comparison of localized lesion at the indicated time-points post-operation.

\begin{tabular}{lccr}
\hline Time & CXL $(\mathrm{n}=10)$ & Stromal injection $(\mathrm{n}=21)$ & P-value \\
\hline 1 week, n (\%) & $9(90.0)$ & $16(76.2)$ & 0.63 \\
2 weeks, n (\%) & $9(90.0)$ & $13(61.9)$ & 0.21 \\
4 weeks, n (\%) & $9(90.0)$ & $9(42.9)$ & 0.02 \\
12 weeks, n (\%) & $9(90.0)$ & $9(42.9)$ & 0.02 \\
24 weeks, n (\%) & $9(90.0)$ & $9(42.9)$ & 0.02 \\
\hline
\end{tabular}

CXL, corneal cross-linking.
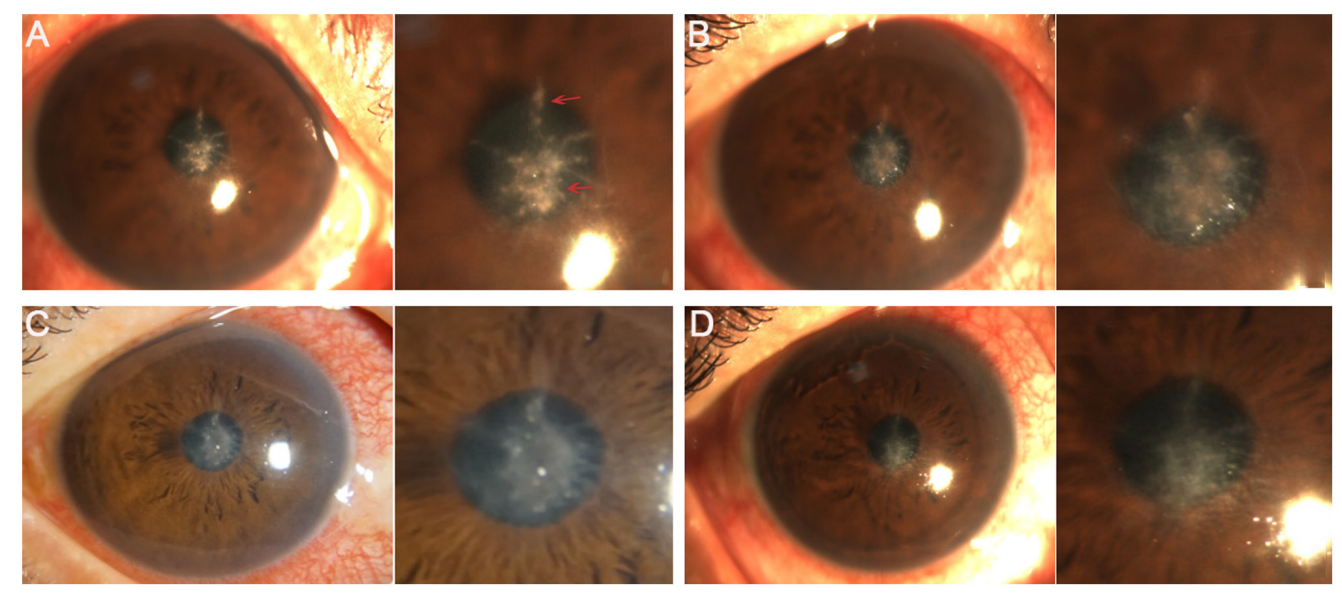

Figure 4. Localized lesions before and after surgery in the corneal cross-linking group. (A) A representative image from a patient before surgery showing an $2 \times 2 \mathrm{~mm}$ ulcer area, with pseudopods (bottom arrow) and satellite foci (top arrow) at the center of the cornea. After surgery, the patient's ulcer surface became clean, the border was gradually cleared and the pseudopods and satellite foci disappeared on days (B) 3, (C) 5 and (D) 14 .

with sodium fluorescein, whilst at 7 days post-surgery, no staining was observed, suggesting that the corneal ulcer was cured. Furthermore, the images of AS-OCT revealed that patients in the CXL group were completely healed 3 months after surgery (Fig. 7). Most patients with fungal keratitis in China are farmers (1). Due to economic conditions, no AS-OCT images were available for the stromal injection group. The association between corneal lesion diameter and healing process is shown in Fig. 8. The corneal epithelium was healed on weeks 4 and 1 after surgery in patients, and the 
Table V. Comparison of ulcer healing rates 1 week after surgery.

\begin{tabular}{lccr}
\hline Status & CXL $(\mathrm{n}=10)$ & Stromal injection $(\mathrm{n}=21)$ & P-value \\
\hline Healing, $\mathrm{n}(\%)$ & $6(60.0)$ & $5(23.8)$ & \\
Not healing, $\mathrm{n}(\%)$ & $4(40.0)$ & $16(76.2)$ & 0.11 \\
Total, $\mathrm{n}(\%)$ & $10(100.0)$ & $21(100.0)$ & \\
\hline
\end{tabular}

CXL, corneal cross-linking.
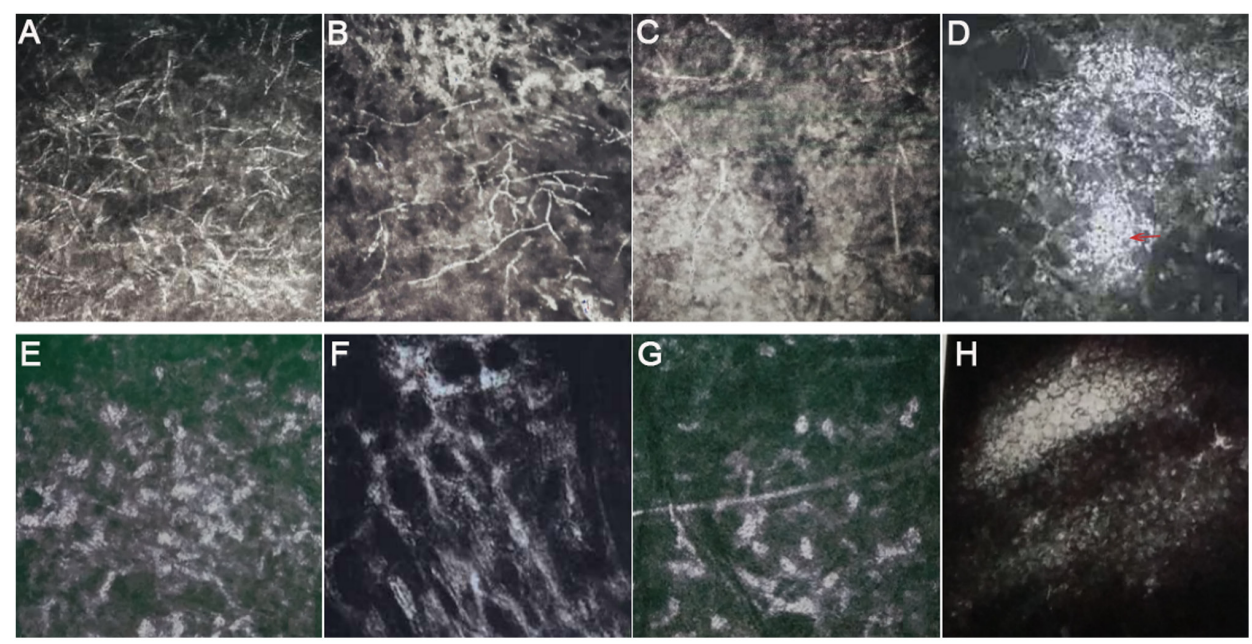

Figure 5. In vivo confocal microscopy images before and after surgery in the corneal cross-linking group. (A-H) These images revealed the scanning depth post-operation was not the same as that pre-operation, which was used to clarify if the hyphae invaded the deep stroma and the infection was controlled. Representative images from different patients showing a large number of fungal mycelia (A), defects in the epithelial tissue (B), irregular cell morphology (C) and inflammatory cell infiltration (arrow) (D) in the shallow stromal layer before operation. (E) The mycelium-like structures in the shallow stromal layer disappeared, whilst the honeycomb structures were observed at 4 weeks postoperatively. (F) The number of inflammatory cells was decreased and a scar was formed at 4 weeks postoperatively. $(\mathrm{G})$ The honeycomb structures in the deep stromal layer were decreased and $(\mathrm{H})$ endothelial cells were regular with normal morphology and arrangement at 4 weeks after surgery (magnification, $\mathrm{x} 400$ ).

Table VI. Comparison of BCVA at 6 months postoperatively ${ }^{\mathrm{a}-\mathrm{c}}$.

\begin{tabular}{lcc}
\hline Time & $\begin{array}{c}\text { CXL } \\
(\mathrm{n}=10)\end{array}$ & $\begin{array}{c}\text { Stromal injection } \\
(\mathrm{n}=21)\end{array}$ \\
\hline Pre-operative BCVA & $1.28 \pm 1.08$ & $1.62 \pm 1.33$ \\
Post-operative BCVA & $0.56 \pm 0.90^{\mathrm{d}}$ & $1.40 \pm 1.35^{\mathrm{e}}$ \\
\hline
\end{tabular}

${ }^{\mathrm{a}} \mathrm{P}=0.22$, group effect; ${ }^{\mathrm{b}} \mathrm{P}<0.0001$, time effect; ${ }^{\mathrm{P}} \mathrm{P}=0.03$, interaction effect; ${ }^{\mathrm{P}}=0.01$, pre-operation vs. post-operation in $\mathrm{CXL}$ group; ${ }^{\mathrm{e}} \mathrm{P}=0.08$, post-operation in CXL group vs. post-operation in stromal injection group. All mixed-model ANOVA. CXL, corneal crosslinking, BCVA, best corrected visual acuity.

lesion diameter was $\geq 5$ and $<5 \mathrm{~mm}$, respectively. This indicated that the healing time of lesions with a diameter $<5 \mathrm{~mm}$ was decreased compared with that of lesions with a diameter $\geq 5 \mathrm{~mm}$.

Visual prognosis. As shown in Table VI, BCVA was not significantly different between the CXL and stromal injection group $(\mathrm{F}=1.58 ; \mathrm{P}=0.22)$, and significant time effect was found $(\mathrm{F}=21.99 ; \mathrm{P}<0.0001)$. There was also significant interaction between the time and surgery method $(\mathrm{F}=5.46 ; \mathrm{P}=0.03)$.
Additionally, the results showed that BCVA was significantly decreased after surgery in the CXL group compared with that before surgery but not in the stromal injection group. BCVA was not significantly different between the CXL and stromal injection group at 6 months postoperatively $(\mathrm{P}=0.08$; Table $\mathrm{VI})$. Furthermore, the percentage of patients $\operatorname{LogMAR}<0.05$ in the CXL group was $80 \%$, but in the stromal injection group was $42.85 \%$, as demonstrated by the BCVA interval distribution at 6 months after surgery. However, there was no obvious difference on visual acuity at postoperative 6 months between the two groups ( $\mathrm{P}=0.22$; Table VII).

Postoperative complications and management. In the CXL group, loss of corneal transparency occurred in one case 4 weeks after surgery (Fig. 9A), whilst one case of corneal thinning was observed in the stromal injection group 3 days postoperatively (Fig. 9B). During follow-up, the patient in the stromal injection group finally underwent corneal transplantation due to poor infection control. However, the infection in the CXL group was controlled when the visual acuity recovered at postoperative 24 weeks. Nevertheless, no other complications were recorded.

Microbiological examination. Prior to surgery, fungal culture results showed that $7(70.0 \%)$ and 16 cases $(76.2 \%)$ were 
Table VII. Interval distribution of BCVA at post-operation in the two groups.

\begin{tabular}{|c|c|c|c|}
\hline BCVA category & $\operatorname{CXL}(n=10)$ & Stromal injection $(n=21)$ & P-value \\
\hline $\log M A R \leq 0.2$ & $5(50.00)$ & $7(33.33)$ & \\
\hline $0.2<\log$ MAR $\leq 0.5$ & $3(30.00)$ & $2(9.52)$ & \\
\hline $0.5<\log \mathrm{MAR} \leq 1.0$ & $0(0.00)$ & $2(9.53)$ & \\
\hline $\log M A R>1.0$ & $2(20.00)$ & $10(47.62)$ & 0.22 \\
\hline
\end{tabular}

CXL, corneal cross-linking, BCVA, best corrected visual acuity; logMAR, logarithm of the minimum angle of resolution.

Table VIII. Comparison of species distribution between the two treatment groups.

\begin{tabular}{lcc}
\hline Species & CXL $(\mathrm{n}=10)$ & Stromal group $(\mathrm{n}=21)$ \\
\hline Fusarium, n (\%) & $4(40.0)$ & $7(33.3)$ \\
Alternaria alternata, $\mathrm{n}(\%)$ & $2(20.0)$ & $4(19.1)$ \\
Aspergillus, $\mathrm{n}(\%)$ & $1(10.0)$ & $2(9.5)$ \\
Candida albicans, $\mathrm{n}(\%)$ & $0(0.0)$ & $1(4.8)$ \\
Other, $\mathrm{n}(\%)$ & $0(0.0)$ & $2(9.5)$ \\
Total, $\mathrm{n}(\%)$ & $7(70.0)$ & $16(76.2)$ \\
\hline
\end{tabular}

CXL, corneal cross-linking.
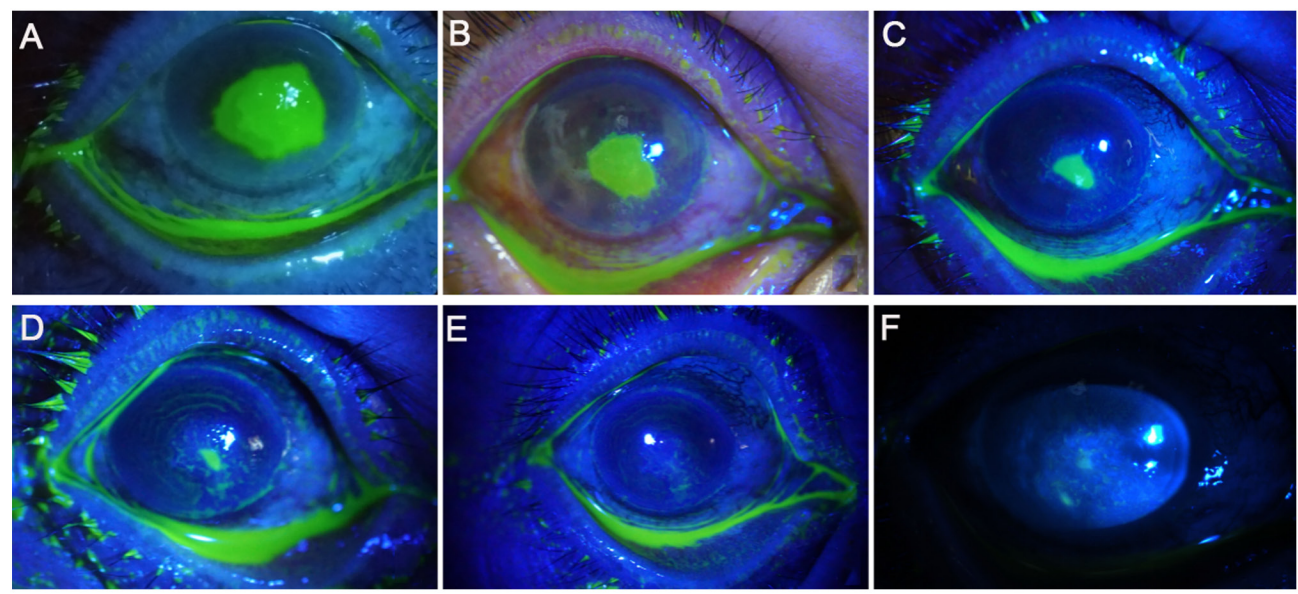

Figure 6. Representative images of ulcer healing 1 week after surgery in the corneal cross-linking group from a patient. (A) A patient's ulcer area in the center of the cornea $(5 \times 5 \mathrm{~mm}$ ) was extensively dyed with sodium fluorescein prior to surgery. Staining of patient's ulcer area with sodium fluorescein on days (B) 2 , (C) 3, (D) 5, (E) 7 and (F) 14 after surgery. (E) Corneal ulcer was not stained from day 7 onwards after surgery, suggesting that the ulcer was cured.

positive for fungal infection in the CXL and stromal injection groups, respectively. Specifically, using strain identification tests, 11 cases of Fusarium, 6 cases of Alternaria alternata, 3 cases of Aspergillus, 1 case of Candida albicans, 1 case of Absidia orchidis and 1 case of Penicillium were identified. However, there was no significant difference in species distribution between the two groups ( $\mathrm{P}>0.99$; Table VIII).

\section{Discussion}

CXL is fast becoming a method of choice for treating ocular surface diseases, including keratoplasty, fungal and bacterial corneal ulcers $(10,13,23)$. CXL controls infection by enhancing the biomechanical strength of the cornea and increasing collagen resistance to enzymatic hydrolysis $(24,25)$. Although the effectiveness of CXL application on fungal corneal ulcers have been previously reported (26), its efficacy on infection control and ulcer healing requires further investigation in a larger population. In the present study, the CXL procedure was improved, following $4 \mathrm{~min}$ irradiation at $30 \mathrm{~mW} / \mathrm{cm}^{2}$ and a total dose of $7.2 \mathrm{~J} / \mathrm{cm}^{2}$, its efficacy was evaluated. The findings suggested that this type of modified CXL conferred superior outcome compared with that by voriconazole in terms of the infection control rate and localized lesions. Furthermore, the cured patients in the CXL group overall exhibited reduced risks for further medication and surgery. 

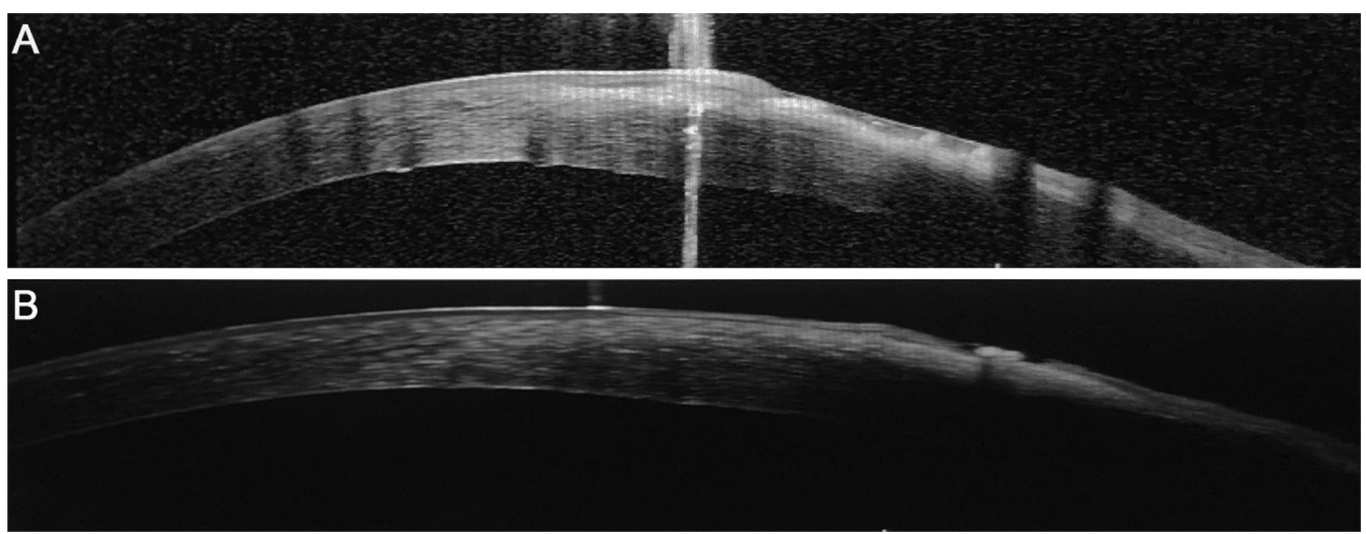

Figure 7. Representative anterior segment optical coherence tomography images. (A) Representative image of cellular stromal infiltration prior surgery. (B) Representative image showing that the corneal epithelium was healed 3 months after surgery (AS-OCT x400).
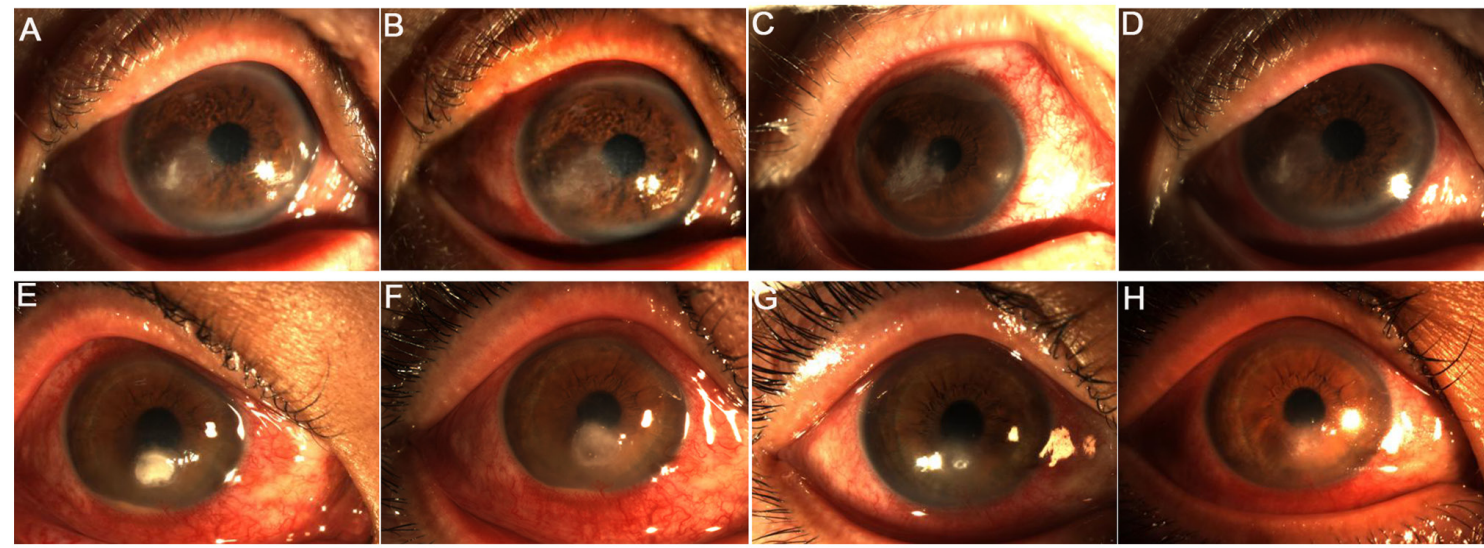

$\mathrm{H}$
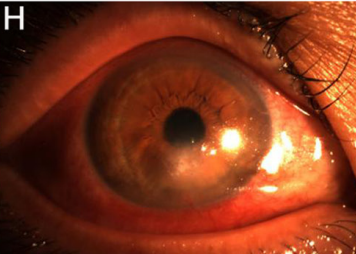

Figure 8. Representative images from patients with different lesion coverage in the corneal cross-linking group. Representative images from a patient with a lesion coverage of $\geq 5 \mathrm{~mm}$ (A) before surgery, and at (B) 1 day, (C) 4 and (D) 12 weeks after surgery, showing the healed epithelium from week 4 onwards postoperatively. Representative images from a patient with a lesion coverage of $<5 \mathrm{~mm}$ (E) before surgery, and at (F) 1 day, (G) 1 and (H) 4 weeks after surgery, showing the healed epithelium from (G) week 1 onwards postoperatively.
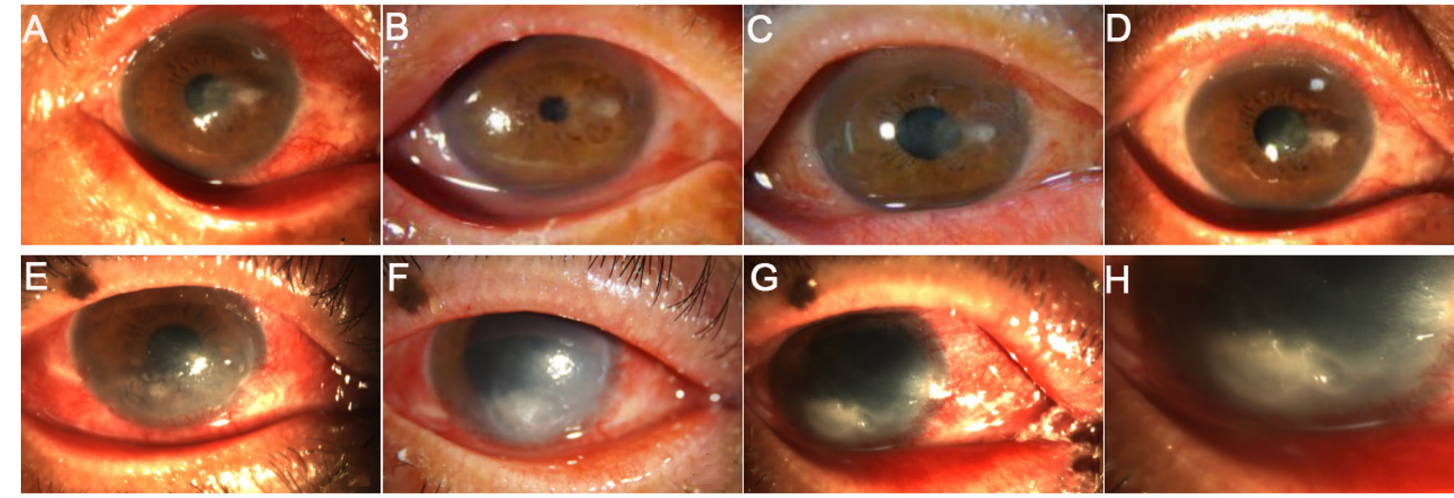

Figure 9. Complications after surgery. The corneal transparency from a patient (A) before surgery, and at (B) 1 day, (C) 1 and (D) 4 weeks after surgery in the corneal cross-linking group, showing that corneal transparency was decreased at 4 weeks after surgery. The corneal thinning from a patient (E) before surgery and at (F) 1 and $(G) 3$ days after surgery in the stromal injection group, showing corneal thinning at day 3 after surgery. (H) Image was close-up image of (G).

FK exists in two forms, the first of which is filamentous fungal keratitis that is most commonly caused by Fusarium and Aspergillus; the second form is yeast keratitis, which results from Candida albicans infection (27). In the present study, microbiological examination demonstrated that Fusarium, Alternaria alternata and Aspergillus, belonging to filamentary fungi, accounted for the majority of fungal distribution. A previous study has revealed that Fusarium and Aspergillus are the most commonly isolated pathogens for FK and account for $>80 \%$ cases in Northern China (4). These findings were consistent with the results of the present study, indicating that filamentous FK was a major cause of severe 
corneal ulcers in China. At present, an increasing number of studies have been focused on the development of effective treatments for fungal corneal ulcer. Hariprasad et al (28) demonstrated that voriconazole could effectively use to treat fungal eye infections. In addition, a number of studies have previously shown that intrastromal voriconazole injection is effective against filamentous fungi infections (29-31). However, other studies have reached the opposite conclusion, suggesting that voriconazole is not sufficient for treating filamentous fungi. Narayana et al (32) indicated that voriconazole was not effective in moderating severe filamentous fungal ulcers. Another similar study in Japan demonstrated in three cases of keratitis caused by Fusarium and Aspergillus that were not completely cured after treatment with voriconazole. Briefly, in corneal infections, fungi are normally observed in the deep stroma, but since voriconazole could not reach Descemet's membrane, infection recurrence occurred in one case. Another patient was treated with topical steroids in the early postoperative stage (33). Regarding topical steroids, Lin et al (34) found a case of a patient who was treated with topical steroids in the early stage and who relapsed after voriconazole treatment, suggesting that treatment ineffectiveness is associated with the use of topical steroids. Furthermore, Cheng et al (35) suggested that failure of voriconazole in the treatment for filamentous fungal keratitis may be associated with the rapid reproduction and mutations of Fusarium induced by the administration of glucocorticoids during the early postoperative period. A similar study revealed that treatment with polyene combined with azole not only reduces the effectiveness of azole against filamentous fungal keratitis, but also enhances drug toxicity (36). In addition, a previous study suggested that not reaching the minimum inhibitory concentration (MIC) for a specific strain is another cause for voriconazole inefficiency against filamentous fungi (37). Specifically, the genus Fusarium consists of $>100$ species, where $\sim 15$ of them may cause corneal infections in humans (38) and exhibit different MIC $(29,39)$, indicating that different Fusarium species is one of the reasons leading to the different conclusions In the present study, 77 eyes $(33.3 \%)$ were temporarily relieved 1 week after surgery, but the infection recurred and the wound was not healed after 2-4 weeks in the stromal injection group. Additionally, two of the four patients who failed to respond to treatment had a history of surgery and medication. The aforementioned findings suggested that voriconazole could be effective for treating filamentous fungal corneal ulcer. However, its administration should be carefully considered for patients with history of surgery and medication and moderate to severe infection.

UV A-riboflavin CXL has been widely used to treat a variety of fungal infections, including Fusarium, Aspergillus and Candida albicans (40). A previous study demonstrated that treatment with 0.1 and $0.25 \%$ riboflavin combined with UV could effectively treat Alternaria alternata infection (41). Another study reported that ulcer healing was achieved in all patients following treatment with traditional CXL, where $\sim 80 \%$ presented significantly improved visual acuity (42). In the present study, the modified CXL procedure with irradiation for $4 \mathrm{~min}$ at $30 \mathrm{~mW} / \mathrm{cm}^{2}$ and a total dose of $7.2 \mathrm{~J} / \mathrm{cm}^{2}$ was used to treat fungal infections. This modified CXL procedure was found to be effective in controlling infection and improving visual acuity. According to the principle that the biological efficacy is associated with total energy dose, Chan et al (17) demonstrated that treatment with increased irradiation of $7-45 \mathrm{~mW} / \mathrm{cm}^{2}$, shortened UV exposure time (from 30 to $5 \mathrm{~min}$ ) and a total dose of $7.2 \mathrm{~J} / \mathrm{cm}^{2}$ was reasonable and safe. A similar study previously revealed that higher fluences of UV-light substantially increased the anti-bacterial efficacy of CXL combined with photoactivated chromophore (43). Therefore, it was hypothesized that the CXL procedure with irradiation for $4 \mathrm{~min}$ at $30 \mathrm{~mW} / \mathrm{cm}^{2}$ and a total dose of $7.2 \mathrm{~J} / \mathrm{cm}^{2}$ for treating patients with fungal corneal ulcer was safe and effective.

Fungal infection is characterized by a large number of hyphae or spores in the focal lesions (44). In the present study, fungal mycelia, defects in the epithelial tissue, irregular cell morphology and inflammatory cell infiltration were observed prior to surgery. However, following CXL therapy, the number of fungal mycelia and inflammatory cell infiltration were decreased and endothelial cells acquired their typical morphology. These findings were consistent with previous studies. Wei et al (9) reported that the symptoms were rapidly relieved in the majority of rabbits with bacterial corneal ulcer, where the area of inflammatory cell infiltration was decreased after treatment with CXL combined with riboflavin and $440 \mathrm{~nm}$ blue light. Additionally, Bamdad et al (23) demonstrated that the defects in endothelial cells were smaller postoperationally compared with those before surgery. Another study also previously showed that re-epithelization occurred in $56 \%$ of patients within 1 week in terms of healing (45), which was consistent with results from the present study, where the rate of ulcer healing 1 week after surgery in the CXL group was $60.0 \%$. The aforementioned findings suggested that treatment with modified CXL accelerated corneal repair and promoted ulcer healing to a certain extent.

Previous studies have suggested that the diameter of corneal lesion is closely associated with the healing process. For example, a prospective study found that the healing time was increased with increasing ulcer and infiltration area (46). Furthermore, Li et al (31) demonstrated that the majority of patients with healed cornea at 1 week postoperatively exhibited corneal lesion diameter $<5.0 \mathrm{~mm}$; however the healing time was $>1$ week in patients with corneal lesion diameter $>5.0 \mathrm{~mm}$. In the present study, the healing time of the epithelium in the subgroup with lesion coverage $<5.0 \mathrm{~mm}$ was shorter compared with that in the $>5.0 \mathrm{~mm}$ subgroup, indicating that corneal lesion diameter was closely associated with the healing process. Furthermore, You et al (47) indicated that lesions with maximum diameters of $<5 \mathrm{~mm}$ could reduce the difficulty of surgery and instability of the cornea. These results suggested that the treatment efficacy of this modified CXL surgery in lesions with coverage $\leq 5.0 \mathrm{~mm}$ could be superior to that in the $\geq 5.0 \mathrm{~mm}$ subgroup.

In the present study, corneal transparency was decreased in one case in the CXL group. Generally, apoptosis occurs to a depth of $<300 \mu \mathrm{m}$ of the corneal stroma $24 \mathrm{~h}$ after surgery, resulting in the loss of corneal transparency $(48,49)$. In the present study, corneal thinning was observed in one case in the stromal injection group. A study also previously showed that cell apoptosis was observed following the surgical removal of the corneal ulcer lesion, leading to the decreased number of 
corneal cells, which in turn resulted in corneal thinning (50). However, no other complications were observed in the present study. The aforementioned data suggested that the modified CXL procedure with irradiation for $4 \mathrm{~min}$ at $30 \mathrm{~mW} / \mathrm{cm}^{2}$ and a total energy dose of $7.2 \mathrm{~J} / \mathrm{cm}^{2}$ was safe and effective for treating fungal corneal ulcers. Nevertheless, this procedure requires to further optimization to relief pain and related complications.

Limitations of the present study included the small sample size. Therefore, a large randomized controlled study should be performed in the future to confirm the aforementioned findings and to explore the risk factors affecting the treatment efficacy and healing rate, including the fungal species and lesion coverage.

In conclusion, modified CXL treatment could result in favorable effects in patients with fungal corneal ulcer regarding the control of infection, localized lesions and accelerated epithelialization. In addition to the reduced risk for complications, including loss of corneal transparency, modified CXL treatment could also minimize the need for surgery.

\section{Acknowledgements}

Not applicable.

\section{Funding}

No funding was received.

\section{Availability of data and materials}

All data generated or analyzed during this study are included in this published article.

\section{Authors' contributions}

YC, MG and LS conceived the study. XM and YC collected materials and samples. YC, XM and LS contributed to data analysis and interpretation of the results. MG and YC provided administrative support. YC and LS confirm the authenticity of all the raw data. All authors read and approved the final manuscript.

\section{Ethics approval and consent to participate}

The present study was approved by the Ethics Committee of General Hospital of Northern Theater Command (approval no. 201736). Written informed consent was obtained from each patient. All procedures were performed in accordance with the Declaration of Helsinki developed by the World Medical Association.

\section{Patient consent for publication}

All patients provided written informed consent for the publication of any associated data and accompanying images.

\section{Competing interests}

The authors declare that they have no competing interests.

\section{References}

1. Wang L, Sun S, Jing Y, Han L, Zhang H and Yue J: Spectrum of fungal keratitis in central China. Clin Exp Ophthalmol 37: 763-771, 2009.

2. Wu J, Zhang WS, Zhao J and Zhou HY: Review of clinical and basic approaches of fungal keratitis. Int J Ophthalmol 9: 1676-1683, 2016.

3. Fini ME, Cook JR and Mohan R: Proteolytic mechanisms in corneal ulceration and repair. Arch Dermatol Res 290 (Suppl 1): S12-S23, 1998.

4. Xie L, Zhong W, Shi W and Sun S: Spectrum of fungal keratitis in north China. Ophthalmology 113: 1943-1948, 2006.

5. Chen Y, Yang W, Gao M, Belin MW, Yu H and Yu J: Experimental study on cryotherapy for fungal corneal ulcer. BMC Ophthalmol 15: 29, 2015.

6. Garg P, Roy A and Roy S: Update on fungal keratitis. Curr Opin Ophthalmol 27: 333-339, 2016.

7. Spoerl E, Mrochen M, Sliney D, Trokel S and Seiler T: Safety of UVA-riboflavin cross-linking of the cornea. Cornea 26: 385-389, 2007.

8. Zhang Y, Conrad AH and Conrad GW: Effects of ultraviolet-A and riboflavin on the interaction of collagen and proteoglycans during corneal cross-linking. J Biol Chem 286: 13011-13022, 2011.

9. Wei S, Zhang C, Zhang S, Xu Y and Mu G: Treatment results of corneal collagen cross-linking combined with riboflavin and $440 \mathrm{Nm}$ blue light for bacterial corneal ulcer in rabbits. Curr Eye Res 42: 1401-1406, 2017.

10. Tal K, Gal-Or O, Pillar S, Zahavi A, Rock O and Bahar I: Efficacy of primary collagen cross-linking with photoactivated chromophore (PACK-CXL) for the treatment of staphylococcus aureus-induced corneal ulcers. Cornea 34: 1281-1286, 2015.

11. Raiskup F and Spoerl E: Corneal crosslinking with riboflavin and ultraviolet A. I. Principles. Ocul Surf 11: 65-74, 2013.

12. Ghanem VC, Ghanem RC and de Oliveira R: Postoperative pain after corneal collagen cross-linking. Cornea 32: 20-24, 2013.

13. Taneri S, Oehler S, Lytle G and Dick HB: Evaluation of epithelial integrity with various transepithelial corneal cross-linking protocols for treatment of keratoconus. J Ophthalmol 2014: 614380, 2014.

14. Lombardo M, Giannini D, Lombardo G and Serrao S: Randomized controlled trial comparing transepithelial corneal cross-linking using iontophoresis with the dresden protocol in progressive keratoconus. Ophthalmology 124: 804-812, 2017.

15. Kim M, Takaoka A, Hoang QV, Trokel SL and Paik DC: Pharmacologic alternatives to riboflavin photochemical corneal cross-linking: A comparison study of cell toxicity thresholds. Invest Ophthalmol Vis Sci 55: 3247-3257, 2014.

16. Gatzioufas Z, Richoz O, Brugnoli E and Hafezi F: Safety profile of high-fluence corneal collagen cross-linking for progressive keratoconus: Preliminary results from a prospective cohort study. J Refract Surg 29: 846-848, 2013.

17. Chan TC, Chow VW, Jhanji V and Wong VW: Different topographic response between mild to moderate and advanced keratoconus after accelerated collagen cross-linking. Cornea 34: 922-927, 2015.

18. Özdemir HB, Kalkancı A, Bilgihan K, Göçün PU, Öğüt B, Karakurt $\mathrm{F}$ and Erdoğan $\mathrm{M}$ : Comparison of corneal collagen cross-linking (PACK-CXL) and voriconazole treatments in experimental fungal keratitis. Acta Ophthalmol 97: e91-e96, 2019.

19. Jorgensen JH, Pfaller MA, Carroll KC, Funke G, Landry ML, Richter SR and Warnock DW (eds): Manual of Clinical Microbiology. 11th edition. American Society for Microbiology, p2892, 2015.

20. Mutoh T, Ishikawa I, Matsumoto Y and Chikuda M: A retrospective study of nine cases of Acanthamoeba keratitis. Clin Ophthalmol 4: 1189-1192, 2010.

21. Karti O,Zengin MO, Cinar E, Tutuncu M, Karahan E, Celik A and Kucukerdonmez C: Effect of 1-and 6-hour-delayed corneal collagen cross-linking on corneal healing in a rabbit alkali-burn model: Clinical and histological observations. Cornea 35: 1644-1649, 2016

22. Sharma S: Diagnosis of infectious diseases of the eye. Eye (Lond) 26 : 177-184, 2012.

23. Bamdad S, Malekhosseini $\mathrm{H}$ and Khosravi A: Ultraviolet A/riboflavin collagen cross-linking for treatment of moderate bacterial corneal ulcers. Cornea 34: 402-406, 2015.

24. Kohlhaas M, Spoerl E, Schilde T, Unger G, Wittig C and Pillunat LE: Biomechanical evidence of the distribution of cross-links in corneas treated with riboflavin and ultraviolet A light. J Cataract Refract Surg 32: 279-283, 2006. 
25. Spoerl E, Wollensak G and Seiler T: Increased resistance of crosslinked cornea against enzymatic digestion. Curr Eye Res 29: 35-40, 2004.

26. Kasparova EA, Sobkova OI and Yang B: Corneal collagen cross-linking in the treatment of infectious keratitis and corneal ulcers. Vestn Oftalmol 133: 113-119, 2017 (In Russian).

27. Lakhundi S, Siddiqui R and Khan NA: Pathogenesis of microbial keratitis. Microb Pathog 104: 97-109, 2017.

28. Hariprasad SM, Mieler WF, Lin TK, Sponsel WE and Graybill JR Voriconazole in the treatment of fungal eye infections: A review of current literature. Br J Ophthalmol 92: 871-878, 2008.

29. Siatiri H, Daneshgar F, Siatiri N and Khodabande A: The effects of intrastromal voriconazole injection and topical voriconazole in the treatment of recalcitrant Fusarium keratitis. Cornea 30: 872-875, 2011

30. Prakash G, Sharma N, Goel M, Titiyal JS and Vajpayee RB Evaluation of intrastromal injection of voriconazole as a therapeutic adjunctive for the management of deep recalcitrant fungal keratitis. Am J Ophthalmol 146: 56-59, 2008.

31. Li SX, Biang J, Li X, Zhang LT and Shi WY: Keratectomy combined with intrastromal injection of voriconazole in treating fungal keratitis. Zhonghua Yan Ke Za Zhi 53: 682-688, 2017 (In Chinese)

32. Narayana S, Krishnan T, Ramakrishnan S, Samantaray PP, Austin A, Pickel J, Porco T, Lietman T and Rose-Nussbaumer J: Mycotic antimicrobial localized injection: A randomized clinical trial evaluating intrastromal injection of voriconazole. Ophthalmology 126: 1084-1089, 2019.

33. Niki M, Eguchi H, Hayashi Y, Miyamoto T, Hotta F and Mitamura Y: Ineffectiveness of intrastromal voriconazole for filamentous fungal keratitis. Clin Ophthalmol 8: 1075-1079, 2014

34. Lin HC, Chu PH, Kuo YH and Shen SC: Clinical experience in managing Fusarium solani keratitis. Int J Clin Pract 59: 549-554, 2005.

35. Cheng J, Zhai HL, Wang JY and Xie LX: Clinical features and treatments of retrocorneal fungal infection. Zhonghua Yan Ke Za Zhi 53: 758-765, 2017.

36. Schacter LP, Owellen RJ, Rathbun HK and Buchanan B: Letter: Antagonism between miconazole and amphotericin B. Lancet 2 : 318, 1976

37. Shen YC, Wang MY, Wang CY, Tsai TC, Tsai HY, Lee HN and Wei LC: Pharmacokinetics of intracameral voriconazole injection. Antimicrob Agents Chemother 53: 2156-2157, 2009.

38. Alastruey-Izquierdo A, Cuenca-Estrella M, Monzón A, Mellado E and Rodríguez-Tudela JL: Antifungal susceptibility profile of clinical Fusarium spp. isolates identified by molecular methods. J Antimicrob Chemother 61: 805-809, 2008.
39. PearsonMM,RogersPD,ClearyJDandChapman SW: Voriconazole: A new triazole antifungal agent. Ann Pharmacother 37: 420-432, 2003.

40. Kashiwabuchi RT, Carvalho FR, Khan YA, Hirai F, Campos MS and McDonnell PJ: Assessment of fungal viability after long-wave ultraviolet light irradiation combined with riboflavin administration. Graefes Arch Clin Exp Ophthalmol 251: 521-527, 2013.

41. Bilgihan K, Kalkanci A, Ozdemir HB, Yazar R, Karakurt F, Yuksel E, Otag F, Karabicak N and Arikan-Akdagli S: Evaluation of antifungal efficacy of $0.1 \%$ and $0.25 \%$ riboflavin with UVA: A comparative in vitro study. Curr Eye Res 41: 1050-1056, 2016.

42. Li Z, Jhanji V, Tao X, Yu H, Chen W and Mu G: Riboflavin/ ultravoilet light-mediated crosslinking for fungal keratitis. Br J Ophthalmol 97: 669-671, 2013.

43. Kling S, Hufschmid FS, Torres-Netto EA, Randleman JB, Willcox M, Zbinden R and Hafezi F: High fluence increases the antibacterial efficacy of PACK cross-linking. Cornea 39: 1020-1026, 2020

44. Wu CS, Wu SS and Chen PC: A prospective study of fungal infection of gastric ulcers: Clinical significance and correlation with medical treatment. Gastrointest Endosc 42: 56-58, 1995.

45. Idrus EA, Utti EM and Mattila JS: Photoactivated chromophore corneal cross-linking (PACK-CXL) for treatment of severe keratitis. Acta Ophthalmol 97: 721-726, 2019.

46. Said DG, Elalfy MS, Gatzioufas Z, El-Zakzouk ES, Hassan MA, Saif MY, Zaki AA, Dua HS and Hafezi F: Collagen cross-linking with photoactivated riboflavin (PACK-CXL) for the treatment of advanced infectious keratitis with corneal melting. Ophthalmology 121: 1377-1382, 2014

47. You X, Li J, Li S and Shi W: Effects of lamellar keratectomy and intrastromal injection of $0.2 \%$ fluconazole on fungal keratitis. J Ophthalmol 2015: 656027, 2015.

48. Wollensak G, Spoerl E, Wilsch M and Seiler T: Keratocyte apoptosis after corneal collagen cross-linking using riboflavin/ UVA treatment. Cornea 23: 43-49, 2004.

49. Linna TU, Vesaluoma MH, Petroll WM, Tarkkanen AH and Tervo TM: Confocal microscopy of a patient with irregular astigmatism after LASIK reoperations and relaxation incisions. Cornea 19: 163-169, 2000

50. Sivak JM and Fini ME: MMPs in the eye: Emerging roles for matrix metalloproteinases in ocular physiology. Prog Retin Eye Res 21: 1-14, 2002. International (CC BY-NC-ND 4.0) License. 